\title{
AVALIAÇÃO DO EFEITO DA HETEROGENEIDADE DO SÓLIDO E DO TAMANHO RELATIVO DAS MOLÉCULAS NA PREDIÇÃO DA ADSORÇÃO MULTICOMPONENTE
}

\author{
L. L. ROMANIELO ${ }^{1}$, e S. ARVELOS ${ }^{1}$ \\ ${ }^{1}$ Universidade Federal de Uberlândia, Faculdade de Engenharia Química \\ E-mail para contato: lucienne@ufu.br
}

\begin{abstract}
RESUMO - A adsorção multicomponente se apresenta como um fenômeno complexo tendo em vista o grande número de variáveis relevantes. Neste trabalho, a heterogeneidade do sólido e o tamanho relativo das moléculas em relação à estrutura do sólido são avaliados. Dados experimentais de isotermas de componentes puros e em misturas, incluindo azeotropismo, foram selecionados para avaliação do efeito das variáveis mencionadas. Os modelos de Solução de Vazios e de Ocupação de Múltiplos Sítios foram aplicados utilizando as modelagens de superfície homogênea e heterogênea. O método da Máxima Verossimilhança foi utilizado para estimação dos parâmetros. De modo geral, a inclusão do efeito da heterogeneidade superficial gerou uma melhoria no desempenho da capacidade preditiva dos modelos apenas para os sistemas no qual o sólido era carvão ativado. Para as zeólitas testadas, a forma homogênea teve melhor desempenho. Os modelos que incluem o tamanho relativo das moléculas apresentaram melhor desempenho.
\end{abstract}

\section{INTRODUÇÃO}

Para o desenvolvimento de processos adsortivos é necessário estabelecer os limites termodinâmicos da adsorção de cada componente presente na mistura em função da condição local de pressão, temperatura e composição. É também necessário fixar as forças motrizes para a transferência de massa nas etapas de adsorção e dessorção dos componentes e calcular os calores isostéricos de adsorção. No entanto, a determinação destas variáveis na maioria das vezes não pode ser realizada a partir de modelos devido à complexidade do fenômeno, a qual pode ser atribuída a vários fatores. Dentre eles, cita-se a não uniformidade da maioria dos sólidos, associada à distribuição de tamanho, forma e energia dos sítios ativos. No intuito de investigar a capacidade de aprimoramento do Modelo de Ocupação de Múltiplos Sítios (MSOM) e do modelo de Solução de Vazios (VSM), o presente trabalho tem como objetivo avaliar a inclusão da heterogeneidade superficial sobre a capacidade preditiva destes modelos.

\section{DESCRIÇÃO DOS MODELOS}

\subsection{Modelo de Ocupação de Múltiplos Sítios}

Forma homogênea: Neste trabalho será utilizado o modelo apresentado por Romanielo et al. (1992), que é uma modificação do modelo originalmente proposto por Nitta et al. (1984), o qual 
considera que as moléculas podem adsorver-se em um ou mais sítios em um sólido homogêneo. O modelo modificado utiliza a aproximação de Guggenheim para contabilizar os estados configuracionais do sistema em substituição da aproximação de Bragg-Williams utilizada por Nitta $e t$ al. (1984). A isoterma do modelo para os componentes puros (Eq. 1) e para misturas (Eq. 2) são escritas:

$$
\begin{aligned}
& P K_{i}=\frac{\theta_{i}\left(1-\beta_{i} \theta_{i}\right)^{(r-1)}}{\left(1-\theta_{i}\right)^{r_{i}}} \exp \left(-\bar{a}_{i} \theta_{i} \frac{u_{i i}}{R T}\right) \\
& y_{i} P K_{i} \phi_{i}=\frac{\theta_{i}\left(1-\sum_{k} \beta_{i} \theta_{k}\right)^{\left(r_{i}-1\right)}}{\left(1-\sum_{k} \theta_{k}\right)^{r_{i}}} \exp \left(-\frac{1}{2} \bar{a}_{i} \sum_{k} \theta_{k} \frac{u_{i k}}{R T}\right) \exp \left(-\frac{1}{2} r_{i} \sum_{k} \frac{\bar{a}_{k}}{R T} \theta_{k} \frac{u_{i k}}{R T}\right)
\end{aligned}
$$

Nas Equações 1 e 2, $P$ representa a pressão total do sistema, $K$ a constante de Henry, $\theta$ $\left(=r_{i} \cdot n_{i} / M\right)$ a fração de cobertura e $\beta\left(=2 .\left(r_{i}-1\right) /(Z . r i)\right)$ é um parâmetro do modelo; $r$ é o número de sítios ocupados por uma molécula, $a$ é a área parcial molar da superfície, $u$ é o parâmetro de interação intermolecular e $\phi$ é o coeficiente de fugacidade; $R$ é a constante universal dos gases, $T$ a temperatura, $Z$ o número de coordenação; $n$ indica a quantidade adsorvida e $M$ é a capacidade máxima de adsorção de um sólido para um dado sistema adsortivo. Os subscritos $i$ e $k$ referem-se aos adsorvatos.

Observa-se que, a princípio, o modelo tem quatro parâmetros a se determinar: $M, r_{i}, K_{i}$ e $u_{i i}$. Porém, como $M$ é uma característica do sólido, o procedimento indicado é a determinação deste parâmetro através da regressão dos dados experimentais de adsorção da menor molécula do sistema, a qual arbitra-se o valor de $r_{i}=1$.

Forma Heterogênea: Romanielo e Krähenbühl (1998) apresentaram a extensão do modelo MSOM para superfícies heterogêneas utilizando a teoria de "patchwise" e função de distribuição de Gauss assimétrica, discretizada em quatro pontos, como proposto por Nitta et al. (1991). Nesta forma, a isoterma local para componentes puros (Eq. 1) é avaliada em todas as regiões do sólido $(m)$ caracterizadas por uma dada energia adsortiva $\left(\varepsilon^{k}\right)$ em termos de uma dada energia de referência $\left(\varepsilon^{0}\right)$ que provê uma constante de Henry de referência $\left(K^{0}\right)$, sendo que a constante de Henry é calculada através da Equação 3.

$$
K^{(m)}=K^{0}\left(1+\frac{\sqrt{2} \sigma}{\varepsilon^{0} / k} t^{(m)}\right)^{-1 / 2} \exp \left(\frac{\sqrt{2} \sigma}{T} t^{(m)}\right)
$$

Na Equação 3 a variância $(\sigma)$ advém da adoção da função densidade de probabilidade para a distribuição de energia adsortiva, $t$ é a energia adimensional e $k$ é a constante de Boltzmann. Os valores de probabilidade $(p)$ adotados em cada seção ou "patch" são aqueles propostos por Nitta et al. (1991). Para a região 1: $\mathrm{t}=0,2735$ e $\mathrm{p}=0,573$; região 2, $\mathrm{t}=0,8230$ e $\mathrm{p}=0,317$; região $3, \mathrm{t}=1,3803$ e $\mathrm{p}=0,097$; região $4, \mathrm{t}=1,9518$ e $\mathrm{p}=0,015$. A quantidade adsorvida $(n)$ é calculada como: 


$$
n=\sum_{k=1}^{m} n^{k}\left(T, P, \varepsilon^{k}\right) p^{k}
$$

\subsection{Modelo de Solução de Vazios (VSM)}

Forma Homogênea: publicado por Suwanayuen e Danner (1980) o modelo VSM trata as fases adsorvida e gasosa como soluções de vazios. O "vazio" é um solvente imaginário ocupando espaços que posteriormente serão ocupados por adsorvatos. Através do critério de igualdade entre os potencias químicos da fase adsorvida e fluida, os autores apresentam a isoterma do modelo:

$$
y_{i} P \phi_{i}=\gamma_{i}^{s} \mathrm{x}_{\mathrm{i}}^{\mathrm{s}} \exp \left(\frac{\Delta \mathrm{G}_{\mathrm{i}}^{0}}{R T}\right) \exp \left(\frac{\pi \bar{a}_{i}}{R T}\right)
$$

Neste modelo, a fração de cobertura de cada componente é dada por $\theta_{1}=n_{1} / M$. A Equação 5 relaciona a fração molar do componente $i$ em fase gasosa, $y_{i}$, com a fração molar adsorvida do componente $i, x_{i}^{s}$, a qual é baseada na quantidade total adsorvida incluindo o vazio $\left(n_{m}^{s, \infty}\right)$. A fração molar experimental, $x_{i}$, é dada pela razão $x_{i}^{\varepsilon} / \theta . \gamma$ representa o coeficiente de atividade. O sobrescrito " $s$ " indica a fase adsorvida e "⿻" indica o valor máximo de adsorção. O subscrito " $m$ " indica que o parâmetro é relativo à mistura (aos adsorvatos e ao vazio).

Conforme demonstrado em Suwanayuen e Danner (1980), a pressão de espalhamento $(\pi)$ relaciona-se com o coeficiente de atividade do vazio $\left(\gamma_{3}{ }^{\mathrm{s}}\right)$ pela Equação 6 em que o subscrito 3 representa o vazio. A energia livre de Gibbs padrão de adsorção $\left(\Delta \mathrm{G}_{\mathrm{i}}{ }^{0}\right)$ é definida como na Equação 7 :

$$
\begin{aligned}
& -\frac{\pi \bar{a}_{i}}{R T}=\left[1+\frac{n_{m}^{s_{0} \infty}-n_{i}^{s_{i} \infty}}{n_{m}^{s}}\right] \ln \left(\gamma_{3}^{s} x_{3}^{s}\right) \\
& \exp \left(\frac{\Delta G_{i}^{0}}{R T}\right)=\frac{1}{K_{i}} n_{i}^{s_{i} \infty} \lim _{x_{i}^{s} \rightarrow 0}\left(\frac{1}{\gamma_{i}^{s}}\right)
\end{aligned}
$$

Assim, observa-se que a forma final da isoterma é dependente do modelo de GE utilizado para contabilizar os coeficientes de atividade dos componentes (adsorvatos e vazio) presentes na fase adsorvida. No modelo VSM original, a equação de Wilson é utilizada para cálculo dos coeficientes de fugacidade nas Equações 5, 6 e 7.

Neste trabalho, foi utilizada a formulação original de Suwanayuen e Danner (1980), porém utilizando o modelo de GE NRTL. Desta forma a isoterma de puro apresenta 5 parâmetros a serem regredidos que são: $K_{i}, n_{i}^{\alpha}$ e $\alpha_{i v}$ (parâmetro de não-aleatoriedade) e $\tau_{1 v}, \tau_{v 1}$ (parâmetros de energia do modelo NRTL). No caso da isoterma de misturas, a interação entre os adsorvatos foi negligenciada. Observa-se que neste modelo a interação adsorvente-adsorvato é ponderada não apenas na constante de Henry $\left(K_{i}\right)$, mas também nos parâmetros de interação do adsorvato e vazio. 
Forma Heterogênea: Nieszporek e Banach (2011) desenvolveram uma forma heterogênea do modelo VSM utilizando o modelo NRTL para substâncias puras. Esta modelagem utiliza várias simplificações. Dentre elas a de que não há interação entre o adsorvato $(i)$ e o vazio $(v)$, nem interação entre dois vazios. Assim, o modelo NRTL apresenta apenas o termo de interação residual do vazio com o adsorvato $\left(\tau_{v 1}\right)$. A forma da isoterma para substâncias puras é então escrita:

$$
P_{1}=\frac{1}{K_{1}}\left(\frac{\theta_{1}}{1-\theta_{1}}\right)^{c} \exp \left\{\frac{\tau_{v 1} \exp \left(-2 \alpha_{1 v} \tau_{v 1}\right)}{\left(1-\exp \left\{-\alpha_{1 v} \tau_{v 1}\right)\left[\theta_{1}+\left(1-\theta_{1}\right) \exp \left\{-\alpha_{1 v} \tau_{v 1}\right\}\right]^{2}\right.}\right\}
$$

Assim, com as simplificações impostas, este modelo apresenta 5 parâmetros sendo $c$ o parâmetro de heterogeneidade, incluído na formulação a partir da proposição de Rudzinski et al. (1994). Para obtenção da extensão ao modelo multicomponente, nós utilizamos a formulação utilizada proposta por Suwanayuen e Danner (1980), de forma que o novo termo para a pressão de espalhamento fica dependente do parâmetro de heterogeneidade :

$$
-\frac{\pi \bar{a}_{i}}{R T}=\left[1+\frac{n_{m}^{s, \infty}-n_{i}^{s, \infty}}{n_{m}^{s}}\right]\left[\ln \left(\gamma_{3}^{s}\right)+c \cdot \ln \left(x_{3}^{s}\right)\right]
$$

\section{METODOLOGIA}

Para avaliar os modelos, bem como a influência dos parâmetros no comportamento de misturas complexas envolvendo azeotrópos, foram selecionados alguns sistemas disponíveis na literatura. A Tabela 1 apresenta a relação das misturas testadas bem como as respectivas referências. Os parâmetros dos componentes puros foram avaliados utilizando-se o método da máxima verossimilhança.

Tabela 1 - Referência bibliográfica e sistemas binários estudados

\begin{tabular}{|c|c|c|c|c|}
\hline Referência & Sólido & Sistema (1)-(2) & $\boldsymbol{T}[\mathbf{K}]$ & $\boldsymbol{P}[\mathbf{k P a}]$ \\
\hline Talu e Ziebel (1986) & Mordenita & Propano-CO & 303 & 41 \\
\hline & & Propano- $\mathrm{H}_{2} \mathrm{~S}$ & 303 & 8 \\
\hline & & $\mathrm{CO}_{2}-\mathrm{H}_{2} \mathrm{~S}$ & 303 & 15 \\
\hline Hyun e Danner (1982) & Zeólita 13X & Isobutano-etileno & 298 e 323 & 138 \\
\hline & & Etileno-CO & 298 e 323 & 138 \\
\hline Yu e Neretnieks (1990) & Carvão ativado & MCH-tolueno & 298 & $1.10^{-5\left(^{*}\right)}$ \\
\hline & & Tolueno-IBMC & 298 & $1.10^{-5\left(^{*}\right)}$ \\
\hline Sakuth et al. $(1998)$ & DAY-13 & Tolueno-1-propanol & 318 & 0,35 e 1,05 \\
\hline & DAY-55 & Tolueno-1-propanol & 318 & 0,35 e 1,05 \\
\hline
\end{tabular}

${ }^{(*)} \mathrm{em}$ atm. $\mathrm{MCH}=$ metil-ciclo-hexano; IBMC = isobutil-metil-cetona

Com os parâmetros dos componentes puros, a adsorção das misturas binárias foi predita, sendo o desvio médio relativo (DMR) utilizado para avaliar o desempenho dos modelos. O DMR é calculado como na Equação 10, em que "med" indica vapor experimental (medido), "cal" o valor calculado e $N p$ o número de pontos experimentais. 


$$
D M R=\frac{1}{N p}\left|\left(\frac{\text { valor }^{\text {med }}-\text { valor }^{\text {cal }}}{\text { valor }^{\text {med }}}\right)\right| * 100 \%
$$

\section{RESULTADOS E DISCUSSÃO}

\subsection{Correlação de Compostos Puros}

A Figura 1 mostra os resultados típicos da correlação dos dados experimentais, apresentados pelos modelos MSOM e VSM nas suas formas homogênea e heterogênea.

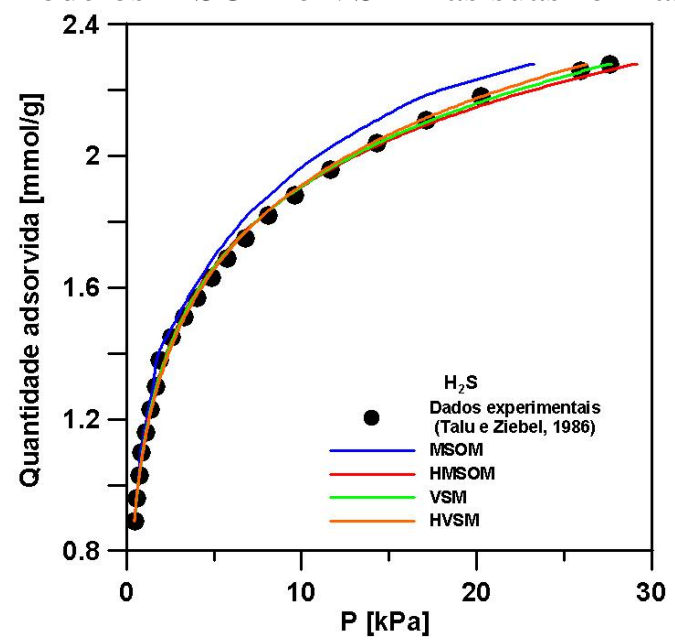

(a)

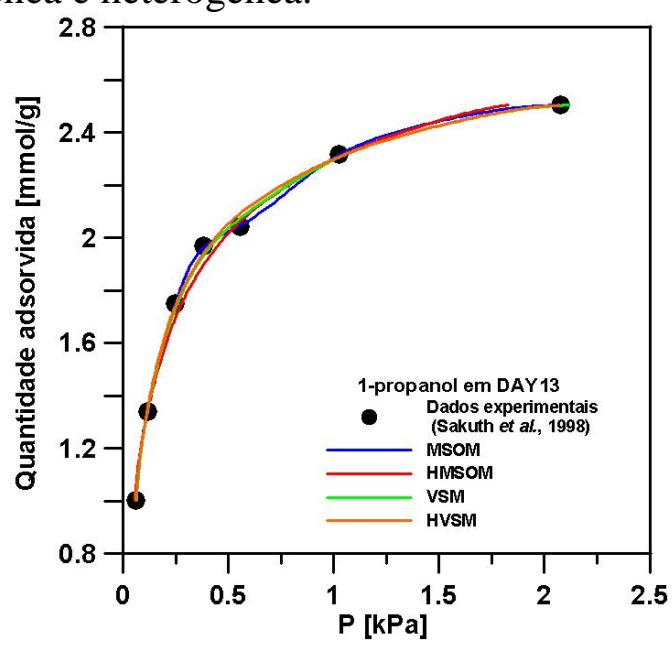

(b)

Figura 1 - Isotermas de adsorção de (a) $\mathrm{H}_{2} \mathrm{~S}$ em mordenita à 303,15 K (dados de Talu e Ziebel, 1986) e de (b) 1-propanol em DAY13 à 318,15 K (dados de Sakuth, 1998).

Observa-se que, de modo geral, todos os modelos representam bem as isotermas dos componentes puros. A Tabela 2 apresenta os parâmetros dos modelos de ocupação de múltiplos sítios nas formas homogênea (MSOM) e heterogênea (HMSOM), enquanto a Tabela 3 apresenta os parâmetros dos modelos de solução de vazios nas formas homogênea (VSM) e heterogênea (HVSM). Os modelos aplicados a superfícies heterogêneas (HMSOM e HVSM) apresentaram desempenho equivalente aos respectivos modelos homogêneos.

\subsection{Correlação de Misturas}

A Tabela 4 apresenta os desvios médios ocorridos na predição da adsorção dos diversos sistemas binários estudados pelos modelos MSOM, HMSOM, VSM e HVSM. De maneira geral, pode-se observar que a inclusão do efeito de heterogeneidade energética no modelo MSOM melhora a predição da quantidade total adsorvida. Tal fato pode ser explicado pela inclusão de dois parâmetros no modelo, favorecendo em especial a correlação dos dados na região de saturação que é responsável pela boa predição da capacidade total adsorvida das misturas.

Quanto aos modelos VSM, verifica-se que o menor desvio obtido na predição dos puros pelos modelos VSM não se confirmou na predição dos binários, nem na forma homogênea, nem 
na forma heterogênea, indicando a falta de representação de efeitos importantes para adsorção altamente não ideal tal como dissimilaridade entre o tamanho das moléculas.

Tabela 2 - Parâmetros para os modelos MSOM estimados para os gases puros

\begin{tabular}{|c|c|c|c|c|c|c|c|c|c|c|}
\hline \multirow[b]{2}{*}{ Adsorvato } & \multicolumn{4}{|c|}{ MSOM } & \multicolumn{6}{|c|}{ HMSOM } \\
\hline & $\begin{array}{c}r_{i} \\
{[-]}\end{array}$ & $\begin{array}{c}K_{i} \\
{\left[\mathrm{P}^{-1}\right]}\end{array}$ & $\begin{array}{l}u / R \\
{[\mathrm{~K}]}\end{array}$ & DMR & $\begin{array}{c}r_{i} \\
{[-]}\end{array}$ & $\begin{array}{l}u / R \\
{[\mathrm{~K}]}\end{array}$ & $\begin{array}{c}K_{i}^{0} \\
{\left[\mathrm{P}^{-1}\right]}\end{array}$ & $\begin{array}{c}\varepsilon_{i}^{0} / k \\
{[\mathrm{~K}]}\end{array}$ & $\sigma_{i}[\mathrm{~K}]$ & DMR \\
\hline Propano & 8,19 & 0,0966 & 55,6 & 1,0 & 4,56 & $-179,7$ & 0,0660 & 1670,4 & 421,9 & 19,6 \\
\hline $\mathrm{CO}_{2}{ }^{\mathrm{a}}$ & 1,00 & 0,0421 & $-6917,3$ & 14,6 & 1,00 & $-1800,6$ & 0,0008 & 2110,4 & 1181,0 & 13,1 \\
\hline $\mathrm{H}_{2} \mathrm{~S}$ & 1,20 & 0,9283 & $-7133,8$ & 11,9 & 1,70 & $-1733,9$ & 0,1610 & 2064,8 & 851,5 & 4,5 \\
\hline Etileno $^{\mathrm{e}}$ & 1,78 & 0,2630 & $-333,8$ & 1,9 & 1,45 & $-246,4$ & 0,0970 & 2146,9 & 471,1 & 13,4 \\
\hline Etileno $^{\mathrm{f}}$ & 1,84 & 0,0630 & $-245,7$ & 0,6 & 1,87 & 446,2 & 0,0191 & 2200,7 & 399,2 & 12,4 \\
\hline Isobutano $^{\mathrm{e}}$ & 2,98 & 5,3099 & $-288,2$ & 2,0 & 2,27 & $-470,7$ & 7,1000 & 682,6 & 31,4 & 28,4 \\
\hline Isobutano ${ }^{\mathrm{f}}$ & 3,58 & 0,5597 & 205,9 & 0,2 & 2,62 & $-425,2$ & 1,8901 & 682,0 & 89,2 & 20,4 \\
\hline $\mathrm{CO}_{2}{ }^{\mathrm{e}}$ & 1,00 & 0,3367 & $-1391,1$ & 1,9 & 1,00 & $-391,9$ & 0,0400 & 1192,5 & 680,1 & 11,3 \\
\hline $\mathrm{CO}_{2}{ }^{\mathrm{f}}$ & 1,00 & 0,0869 & $-1406,6$ & 0,3 & 1,00 & $-535,3$ & 0,0150 & 1710,7 & 630,0 & 6,7 \\
\hline Tolueno ${ }^{b}$ & 1,40 & 0,0601 & $-7626,5$ & 21,5 & 1,20 & $-924,8$ & 1,3030 & 456,9 & 124,6 & 14,2 \\
\hline IBMC & 1,00 & 0,1134 & $-17202,7$ & 21,7 & 1,00 & $-1653,4$ & 1,4940 & 1055,8 & 309,0 & 7,0 \\
\hline $\mathrm{MCH}$ & 1,96 & 0,0943 & $-9999,0$ & 23,3 & 1,45 & $-1362,9$ & 1,0790 & 2926,7 & 257,8 & 3,6 \\
\hline Tolueno $^{c}$ & 1,40 & 30,2260 & $-154,1$ & 1,5 & 1,46 & $-290,2$ & 6,6500 & 987,6 & 769,2 & 6,6 \\
\hline Propano $^{c}$ & 1,00 & 15,8657 & $-422,0$ & 1,1 & 1,00 & $-560,2$ & 3,2612 & 1500,6 & 659,2 & 6,2 \\
\hline Tolueno $^{\mathrm{d}}$ & 1,49 & 38,9674 & $-360,9$ & 2,0 & 1,61 & $-105,5$ & 3,7960 & 1807,6 & 672,8 & 1,8 \\
\hline Propanol $^{\mathrm{d}}$ & 1,00 & 1,1783 & 482,5 & 4,6 & 1,00 & $-200,2$ & 0,2670 & 1506,1 & 667,2 & 3,3 \\
\hline
\end{tabular}

${ }^{\mathrm{a}}$ Adsorvido em mordenita; ${ }^{\mathrm{b}} \mathrm{em}$ carvão, ${ }^{\mathrm{c} e m ~ D A Y 13, ~}{ }^{\mathrm{d}} \mathrm{em}$ DAY55, ${ }^{\mathrm{e}} \mathrm{T}_{1}=298,15 \mathrm{~K},{ }^{\mathrm{f}} \mathrm{T}_{2}=323,15 \mathrm{~K}$; MSOM: M=15,00 em mordenita, 6,15 em 13X à $\mathrm{T}_{1}, 6,52 \mathrm{em} \mathrm{13X}$ à $\mathrm{T}_{2}, 50,99 \mathrm{em}$ carvão, $2,75 \mathrm{em}$ DAY13, 2,89 mmol/g em DAY55; HMSOM: $\mathrm{M}=7,84 \mathrm{em}$ mordenita, 4,64 em 13X à $\mathrm{T}_{1}, 5,01 \mathrm{em} 13 \mathrm{X}$ à $\mathrm{T}_{2}, 4,28 \mathrm{em}$ carvão, 3,00 em DAY13, 3,23 mmol/g em DAY55

Quanto ao modelo HVSM, para que algumas misturas binárias não foi possível obter uma solução dentro dos limites físicos da adsorção. Tal fato pode estar associado a vários fatores dentre aqueles associados à estimação paramétrica e as simplificações adotadas no modelo. A Figura 2 apresenta os resultados da predição da fração molar adsorvida para 2 sistemas binários.

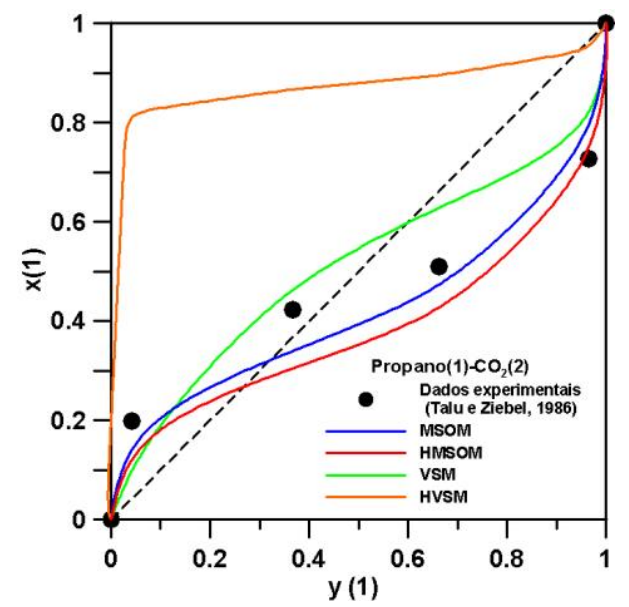

(a)

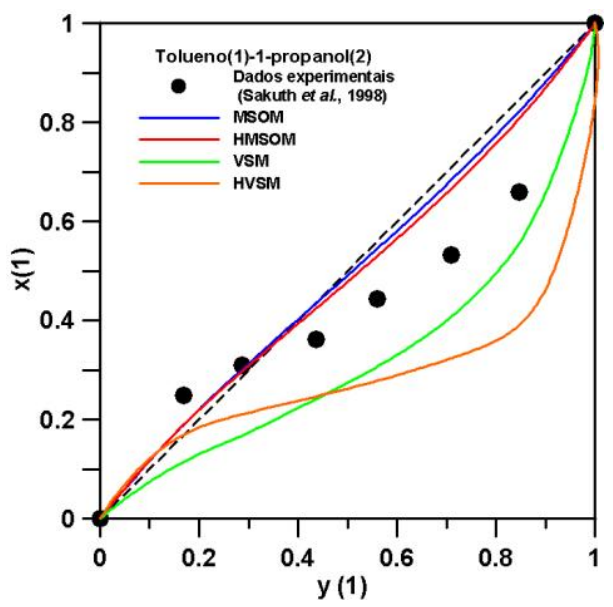

(b)

Figura 2. Predição das frações molares adsovidas dos sistemas (a)- Propano- $\mathrm{CO}_{2}$ em H-mordenita e (b)-Tolueno-propanol em DAY13. 
Tabela 3 - Parâmetros para os modelos VSM estimados para os gases puros

\begin{tabular}{|c|c|c|c|c|c|c|c|c|c|c|c|c|}
\hline \multirow[b]{2}{*}{ Adsorvato } & \multicolumn{6}{|c|}{ VSM } & \multicolumn{6}{|c|}{ HVSM } \\
\hline & $\begin{array}{c}K_{i} \\
{\left[\mathrm{P}^{-1}\right]}\end{array}$ & $\begin{array}{c}M \\
{[\mathrm{mmol} / \mathrm{g}]}\end{array}$ & $\begin{array}{l}\tau_{v l} \\
{[-]}\end{array}$ & $\begin{array}{l}\tau_{l v} \\
{[-]}\end{array}$ & $\begin{array}{l}\alpha_{i v} \\
{[-]}\end{array}$ & DMR & $\begin{array}{c}K_{i} \\
{\left[\mathrm{P}^{-1}\right]}\end{array}$ & $\begin{array}{c}M \\
{[\mathrm{mmol} / \mathrm{g}]}\end{array}$ & $\begin{array}{l}\tau_{v l} \\
{[-]}\end{array}$ & $\begin{array}{c}c_{i} \\
{[-]}\end{array}$ & $\begin{array}{l}\alpha_{i v} \\
{[-]}\end{array}$ & DMR \\
\hline Propano & 1,1786 & 1,2586 & 5,40 & $-20,1$ & 0,37 & 9,9 & 11,8117 & 1,7331 & $-3,03$ & 1,08 & 0,24 & 16,5 \\
\hline $\mathrm{CO}_{2}{ }^{\mathrm{a}}$ & 0,6022 & 3,1416 & $-1,18$ & 1,19 & 0,1 & 11,0 & 0,0038 & 5,9991 & 2,50 & 2,86 & 0,60 & 2,5 \\
\hline $\mathrm{H}_{2} \mathrm{~S}$ & 3,3548 & 2,3666 & 5,78 & $-2,75$ & 0,23 & 3,3 & 0,1759 & 5,8477 & $-2,38$ & 2,62 & 0,39 & 4,7 \\
\hline Etileno $^{\mathrm{e}}$ & 0,3312 & 3,1121 & 5,22 & $-1,98$ & 0,21 & 7,12 & 5,1881 & 3,2611 & 1,97 & 1,16 & 0,25 & 6,6 \\
\hline Etileno $^{\mathrm{f}}$ & 0,6204 & 2,831 & 0,62 & 2,21 & 0,15 & 5,15 & 22,3835 & 2,9673 & $-0,24$ & 1,19 & 0,18 & 6,9 \\
\hline Isobutano $^{\mathrm{e}}$ & 18,265 & 2,1161 & $-6,43$ & 9,12 & 0,11 & 17,9 & 16,0420 & 2,6238 & $-3,90$ & 1,69 & 0,36 & 23,2 \\
\hline Isobutano ${ }^{\mathrm{f}}$ & 0,1800 & 2,6288 & 4,77 & $-2,83$ & 0,15 & 12,8 & 7,0181 & 1,8087 & 6,38 & 2,68 & 0,20 & 15,1 \\
\hline $\mathrm{CO}_{2}{ }^{\mathrm{e}}$ & 3,3944 & 5,7885 & $-4,27$ & 1,19 & 0,36 & 5,98 & 8,1292 & 5,2013 & 1,71 & 2,37 & 0,18 & 7,4 \\
\hline $\mathrm{CO}_{2}{ }^{\mathrm{f}}$ & 0,1667 & 4,1866 & $-0,88$ & 0,41 & 0,65 & 4,37 & 0,4268 & 5,2027 & $-0,82$ & 1,26 & 0,40 & 5,6 \\
\hline Tolueno ${ }^{b}$ & 9,25 & 3,3303 & 24,38 & $-12,0$ & 0,04 & 9,3 & 0,2723 & 8,7205 & 1,74 & 3,31 & 0,23 & 10,7 \\
\hline IBMC & 22,49 & 38,6559 & $-3,54$ & 3,47 & 0,41 & 4,2 & 0,2582 & 8,9122 & 0,25 & 2,89 & 0,28 & 15,5 \\
\hline $\mathrm{MCH}$ & 15,35 & 3,5330 & $-2,66$ & 1,11 & 0,30 & 4,8 & 0,1776 & 5,5871 & 0,45 & 3,13 & 0,29 & 4,1 \\
\hline Tolueno $^{c}$ & 3,4928 & 1,9510 & 3,05 & $-3,07$ & 0,16 & 10,6 & 18,9304 & 2,3825 & $-7,86$ & 1,51 & 0,57 & 10,3 \\
\hline Propanol $^{\mathrm{c}}$ & 1,2600 & 2,8217 & 9,32 & $-6,59$ & 0,05 & 4,6 & 8,0355 & 3,9075 & $-8,63$ & 1,19 & 0,39 & 3,5 \\
\hline Tolueno $^{\mathrm{d}}$ & 0,0678 & 1,8729 & 10,40 & $-1,31$ & 0,18 & 21,8 & 16,0928 & 2,2663 & $-10,48$ & 1,58 & 0,47 & 8,6 \\
\hline Propanol $^{\mathrm{d}}$ & 3,6129 & 2,8069 & 0,16 & 0,78 & 0,33 & 6,4 & 5,6867 & 3,6747 & $-2,68$ & 1,64 & 0,36 & 7,7 \\
\hline
\end{tabular}

Tabela 4 - Correlação de misturas utilizando os modelos MSOM e VSM

\begin{tabular}{|c|c|c|c|c|c|c|c|c|}
\hline \multirow[b]{2}{*}{ Mistura (1)-(2) } & \multicolumn{2}{|c|}{ MSOM } & \multicolumn{2}{|c|}{ HMSOM } & \multicolumn{2}{|c|}{ VSM } & \multicolumn{2}{|c|}{ HVSM } \\
\hline & $\begin{array}{c}\mathrm{DMR} \\
x_{1}\end{array}$ & $\begin{array}{c}\text { DMR } \\
n^{*}\end{array}$ & $\begin{array}{c}\mathrm{DMR} \\
x_{1}\end{array}$ & $\begin{array}{c}\text { DMR } \\
n^{*}\end{array}$ & $\begin{array}{c}\mathrm{DMR} \\
x_{1}\end{array}$ & $\underset{n^{*}}{\mathrm{DMR}}$ & $\begin{array}{c}\mathrm{DMR} \\
x_{1}\end{array}$ & $\underset{n^{*}}{\operatorname{DMR}}$ \\
\hline Propano- $\mathrm{CO}_{2}$ & 12,8 & 10,3 & 12,1 & 6,4 & 24,70 & 24,07 & 57,51 & 21,71 \\
\hline Propano- $\mathrm{H}_{2} \mathrm{~S}$ & 9,6 & 14,9 & 31,0 & 13,9 & 54,92 & 25,00 & 111,1 & 104,0 \\
\hline $\mathrm{CO}_{2}-\mathrm{H}_{2} \mathrm{~S}$ & 18,7 & 4,6 & 17,9 & 6,5 & 137,03 & 40,69 & 22,2 & 47,1 \\
\hline Isobutano-etileno $^{\mathrm{a}}$ & 12,2 & 3,2 & 12,7 & 2,6 & 23,21 & 43,84 & $-{ }^{g}$ & - \\
\hline Etileno- $\mathrm{CO}_{2}{ }^{\mathrm{a}}$ & 8,2 & 5,5 & 16,5 & 0,6 & 22,21 & 4,76 & - & - \\
\hline Isobutano-etileno $^{\mathrm{b}}$ & 4,7 & 3,3 & 29,6 & 6,1 & 17,00 & 27,66 & - & - \\
\hline Etileno- $\mathrm{CO}_{2}{ }^{\mathrm{b}}$ & 7,9 & 7,0 & 15,1 & 3,8 & 31,50 & 11,78 & - & - \\
\hline MCH-tolueno & 8,7 & 28,7 & 6,7 & 5,6 & 43,34 & 11,78 & - & - \\
\hline Tolueno-IBMC & 6,4 & 22,8 & 9,4 & 3,9 & 73,53 & 13,29 & - & - \\
\hline Tolueno-Propanol $^{\mathrm{c}}$ & 26,1 & 9,3 & 24,4 & 12,3 & 31,1 & 13,0 & 35,3 & 16,1 \\
\hline Tolueno-Propanol $^{\mathrm{d}}$ & 20,3 & 4,5 & 18,62 & 4,1 & 37,2 & 15,5 & 35,5 & 28,0 \\
\hline Tolueno-Propanol $^{\mathrm{e}}$ & 24,8 & 14,9 & 19,1 & 15,3 & 33,6 & 21,2 & 55,6 & 17,4 \\
\hline Tolueno-Propanol $^{\mathrm{f}}$ & 19,1 & 9,1 & 17,7 & 8,9 & 35,9 & 17,4 & 60,6 & 33,8 \\
\hline
\end{tabular}

Embora os modelos MSOM, HMSOM e VSM tenham predito a inversão de fases observada para os sistemas reportados, os modelos MSOM apresentaram, na média, um menor desvio na predição tanto da fração molar adsorvida quanto da quantidade total adsorvida. Tal fato indica que o tamanho relativo das moléculas é uma variável muito importante na inversão da seletividade. 


\section{9 a 22 de outubro de 2014 \\ Florianópolis/SC}

\section{CONCLUSÃO}

Pelos resultados observados pode-se concluir que:

- Apesar dos modelos de Solução de Vazios (VSM) testados apresentarem um maior número de parâmetros, os mesmos não apresentaram uma melhoria significativa em relação à correlação dos componentes puros. O desenvolvimento do modelo HVSM necessita ainda de estudo paramétrico;

- Os modelos MSOM apresentaram, de modo geral, uma melhor predição das frações molares e quantidades adsorvidas para as misturas testadas. Tal fato indica a grande importância da variável que é tamanho relativo das moléculas.

\section{REFERENCIAS}

HAGHTALAB, A.; FARZAD, S. A new gas adsorption isotherm using the vacancy solution theory and NRTL coefficient model. Fluid Phase Equilib., v. 292, p. 36-41, 2010.

HYUN, S. H.; DANNER, R. P. Equilibrium adsorption of ethane, ethylene, isobutane, carbon d oxide and their mixtures. J. Chem Eng. Data, v. 27, p. 196-200, 1982.

MUNAKATA, K. Vacancy solution model formulated by NRTL equation for correlation of adsorption equilibria. J. Chem. Eng. Jpn., v. 40, p. 398-407, 2007.

NIESZPOREK, K.; BANACH, T. The improved NRTL adsorption isotherm for the case of energetically heterogeneous solid surfaces. Fluid Phase Equilib., v. 306, p. 212-218, 2011.

NITTA, T.; SHIGETOMI, T; KURO-OKA, M.; KATAYAMA, T. An adsorption isotherm of multi-site occupancy model for homogeneous surface. J. Chem. Eng. Jpn., v. 17, p. 39-45, 1984.

NITTA, T.; YAMAGUCHI, A.; TOKUNAGA, N..; KATAYAMA, T. A practical isotherm equation for adsorption on a heterogeneous surface and its applications to single and mixed gas adsorption on an activated carbon fiber. J. Chem. Eng. Jpn., v. 24, p. 312-319, 1991.

ROMANIELO, L. L.; KRÄHENBÜHL, M. A. A multi-site occupancy model for adsorption on heterogeneous surfaces. Anais $6^{\text {th }}$ International Conference on Fundamentals of Adsorption, p. 5, Paris, França, 1998.

ROMANIELO, L. L.; TAVARES F. W.; KRISHNASWAMY, R. A simple multi-site occupancy model for multicomponent adsorption isotherms. Anais III Equifase, p. 701, Oaxaca, México, 1992.

RUDZINSKI, W.; NIESZPOREK, K.; MOON, H.; RHEE, H. K. Fundamentals of mixed gas adsorption on heterogeneous solid surfaces, Heterogeneous Chem. Rev., vol. 1, p. 275-308, 1994.

SAKUTH, M.; MEYER, J.; GMEHLING, J. Measurement and prediction of binary adsorption equilibria of vapors on dealuminated Y-zeolites (DAY). Chem. Eng. Process., v. 37, p. 267-277, 1998.

SUWANAYUEN, S.; DANNER. R. P. A gas adsorption isotherm equation based on vacancy solution theory. AIChE J., v. 26, p.68-76, 1980.

TALU, O.; ZWIEBEL, I. Multicomponent adsorption equilibria of nonideal mixtures. AIChE J., vol. 32, p. 1263-1270, 1986.

YU, J.; NERETNIEKS, I. Single component and multicomponent adsorption equilibria on activated carbon of methilcyclohexane, toluene, and isobuthyl methyl ketone. Ind. Eng. Chem. Res., v. 29, p. 220$231,1990$.

\section{Agradecimentos}

Os autores agradecem à FAPEMIG (Processo PCE-00089-14) pelo apoio concedido 